\title{
Validation of the spanish version of the multiple sclerosis international quality of life (musiqol) questionnaire
}

Oscar Fernández ${ }^{1 *}$, Victoria Fernández², Karine Baumstarck-Barrau ${ }^{3}$ Luis Muñoz ${ }^{1}$ Maria del Mar Gonzalez Alvarez ${ }^{1}$

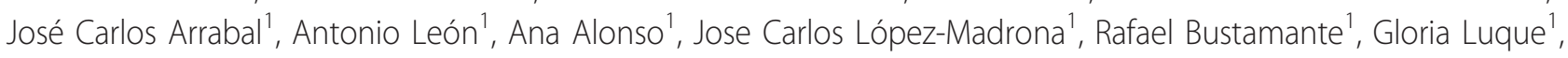
Miguel Guerrero ${ }^{2}$, Elisabetta Verdun di Cantogno ${ }^{4}$ and Pascal Auquier ${ }^{3}$, for the MusiQoL study group of Spain

\begin{abstract}
Background: The Multiple Sclerosis International Quality Of Life (MusiQoL) questionnaire, a 31-item, multidimensional, self-administrated questionnaire that is available in 14 languages including Spanish, has been validated using a large international sample. We investigated the validity and reliability of the Spanish version of MusiQoL in Spain.

Methods: Consecutive patients with different types and severities of multiple sclerosis (MS) were recruited from 22 centres across Spain. All patients completed the MusiQoL questionnaire, the 36-Item Short Form (SF-36) health survey, and a symptoms checklist at baseline and 21 days later. External validity, internal consistency, reliability and reproducibility were tested.

Results: A total of 224 Spanish patients were evaluated. Dimensions of MusiQoL generally demonstrated a high internal consistency (Cronbach's alpha: 0.70-0.92 for all but two MusiQoL domain scores). External validity testing revealed that the MusiQoL index score correlated significantly with all SF-36 dimension scores (Pearson's correlation: 0.46-0.76), reproducibility was satisfactory (intraclass correlation coefficient: 0.60-0.91), acceptability was high, and the time taken to complete the 31-item questionnaire was reasonable (mean [standard deviation]: 9.8 [11.8] minutes).

Conclusions: The Spanish version of the MusiQoL questionnaire appears to be a valid and reliable instrument for measuring quality of life in patients with MS in Spain and constitutes a useful instrument to measure health-related quality of life in the clinical setting.
\end{abstract}

\section{Background}

Health-related quality-of-life (HRQoL) measurements have become an important instrument in population health assessment, treatment evaluation and care management [1-3]. HRQoL indicators depend on the completion of a well-validated questionnaire that assesses health status in individual patients as perceived by themselves through the physical, mental, social and behavioural components of well-being and function [4]. Thus, one major challenge in elaborating the content of

\footnotetext{
* Correspondence: med001832@saludalia.com 'Institute of Clinical Neurosciences. Service of Neurology. Hospital Regional Universitario Carlos Haya, Avda. Carlos Haya s/n, 29010 Málaga, Spain Full list of author information is available at the end of the article
}

the HRQoL dimensions to be measured is to ensure that patients' perceptions are accurately taken into account [5]. Specific HRQoL questionnaires that are available for MS in Spain are FAMS (Functional Assessment of Multiple Sclerosis) [6] and MSQOL-54 (Multiple Sclerosis Quality of Life 54) [7]. However, both of these are based on generic QoL instruments.

The Multiple Sclerosis International Quality of Life (MusiQoL) questionnaire, a specific, self-administered, multidimensional questionnaire, was co-developed and initially validated in 15 countries including Spain [8]. The initial item pool of the Functional Assessment of Multiple Sclerosis included items from the general version of the Functional Assessment of Cancer Therapy quality of

\section{Biomed Central}


life instrument. The other items were generated by patients, providers, and literature review. The MSQOL54 has been adapted from the SF36 measure by the addition of five unchanged SF36 scales, three "altered" SF36 scales (one item added to each scale), and five new scales incorporated 15 additional items. Modifying existing measures by adding MS-specific items was not found to be as useful. The content of the QoL questionnaires is now well-recognized as more appropriate when it is derived from the patients' point of view/patients' interviews. MusiQoL questionnaire specifically reflects the point of view of patients with MS on the impact of the disease on their daily life and is anchored in an explicit conceptual approach as recommended by McKenna [5]. We investigated the validity and reliability of the Spanish version of MusiQoL in Spain.

\section{Methods \\ Patients}

The patients enrolled in the validation study consisted of inpatients and outpatients who were followed in an international multicentre study coordinated by an international steering committee (15 senior neurologists, two experts in HRQoL, one expert in health economics and two external advisors). The inclusion criteria were a diagnosis of MS according to Poser [9] or McDonald [10] criteria as the main diagnosis for more than 6 months, age older than 18 years, informed consent to participate in the study and Spanish as the native language. The main exclusion criteria included a main diagnosis other than MS, dementia, current severe relapses, inability to complete the questionnaire independently and withdrawal of consent. Patients were enrolled between January 2004 and February 2005 from 22 centres distributed throughout Spain.

\section{Study design}

Patients were evaluated at inclusion and re-tested a mean (standard deviation) of 21 (7) days later. The self-administered survey materials that were completed by the patients included the MusiQoL questionnaire, one checklist of 14 symptoms reported by patients suffering from MS (e.g. lack of sensation in touch, lack of sensation in position, fatigue, visual problems, urinary incontinence) and the 36-Item Short Form (SF-36) health survey [11].

The SF-36 is the most widely used generic QoL scale in MS, consisting of 36 items that describe eight dimensions: Physical Functioning (PF), Social Functioning (SF), Role-Physical Problems (RPP), Role-Emotional Problems (REP), Mental Health (MH), Vitality (VIT), Bodily Pain (BP), and General Health (GH). Each dimension is scored from 0-100; the higher the score, the better the perceived state of health.
MusiQoL comprises 31 items that describe nine dimensions (Additional file 1 and Additional file 2). Each dimension is named according to its constitutive items, as follows: activities of daily living (ADL, 8 items), psychological well-being (PWB, 4 items), symptoms (SPT, 3 items), relationships with friends (RFr, 4 items), relationships with family ( $R F a, 3$ items), relationship with the healthcare system (RHCS, 3 items), sentimental and sexual life (SSL, 2 items), coping (COP, 2 items) and rejection (REJ, 2 items). Each item was answered using a sixpoint Likert scale, where $1=$ 'never/not at all', 2 = 'rarely/ a little', 3 = 'sometimes/somewhat', 4 = 'often/a lot', 5 = 'always/very much', 6 = 'not applicable'. The negatively worded item scores were reversed so that higher scores indicated a higher level of HRQoL. For each patient, the score of each dimension was obtained by computing the mean of the item scores of the dimension. If fewer than half of the items were missing, the mean of the non-missing items was substituted for the missing items. All dimension scores were linearly transformed to a $0-100$ scale. A global index score was computed as the mean of the dimension scores.

In addition, an experienced local neurologist collected sociodemographic data, clinical history (related or unrelated to MS), and information on the type of treatment for MS. The neurologist also rated the following: Poser classification (or McDonald classification), the Expanded Disability Status Scale (EDSS) [12], the Ambulation Index (AI) for MS [13], the Folstein Mini-Mental State Examination (MMSE) [14] and the Clinical Global Impression (CGI) of severity scale (mild, moderate, severe) [15]. A detailed physical examination was conducted at baseline and any abnormalities in the organ systems inspected were noted.

At re-testing, patients completed the same questionnaire and one additional question assessing changes in health status. Neurologists collected data on current care and treatment of MS, rated the scores for the EDSS and CGI, and assessed patient health status compared with that reported at the initial inclusion stage (i.e. worsened, remaining stable, improved).

\section{Statistical analyses}

The linguistic transcultural equivalence was ensured by the codevelopment process, including interview and selection of items conducted in Spain.

The multidimensional structure (construct validity) of the Spanish version of the MusiQoL questionnaire was checked using multitrait/multi-item analysis program (MAP) [16] analyses. Internal structural validity was assessed using item-dimension correlations: item internal consistency (IIC) was assessed by correlating each item with its scale (correlation of 0.4 for supporting item 
internal consistency [IIC]) and item discriminant validity (IDV) was assessed by determining the extent to which items correlates with the dimension they are hypothesized to represent than with the other ones. Floor and ceiling effects were assessed by the homogeneous repartition of the response distribution. For each dimension, internal consistency reliability was assessed by Cronbach's alpha coefficient. A Cronbach's alpha coefficient of at least 0.7 was expected for each scale [17]. The unidimensionality of each scale was assessed using Rasch analyses: item goodness-of-fit statistics (INFIT) and coefficient of Loevinger $(\mathrm{H})$. INFIT ranging between 0.7 and 1.2 and $\mathrm{H}$ of at least 0.40 ensure that all the items of the scale tend to measure the same concept.

To explore external validity, relations between: i) dimensions of MusiQoL and the SF-36, and ii) dimensions of MusiQoL and the 14-symptom scale assessing miscellaneous domains, were examined using Pearson's correlation coefficients $(r)$. The underlying assumption was that MusiQoL dimension scores would be more correlated with the scores of similar dimensions from the other instruments than with the scores of dissimilar dimensions [8]. The discriminant validity was determined by assessing the associations between the MusiQoL dimension scores and sociodemographic and clinical features. For qualitative variables, mean dimension scores of the MusiQoL were compared across patient groups that were expected to differ (e.g. MS forms, CGI, gender, family status, educational level, employment status, housing) using one-way analysis of variance. Quantitative variables (e.g. MMSE, AI, EDSS score, age) were analysed using Pearson's correlation coefficients.

Reproducibility was tested through test-re-test reliability using intraclass correlation coefficients (ICC) between the two successive assessments in patients with stable clinical disease. Stable clinical disease was defined using both the physician clinical global impression and the EDSS.
Acceptability was assessed by calculating the average time of completion of the MusiQoL questionnaire and the percentage of missing data per dimension.

Data analyses were performed using SPSS 11.0, MAP$\mathrm{R}$, and WINSTEP software.

\section{Results}

\section{Patient characteristics}

The study sample included 224. Spanish patients with MS (Table 1). The mean (standard deviation [SD]) age was 39 (10) years $(\min =20 / \max =63)$. More than $75 \%$ of patients had clinically definite MS according to Poser criteria. Almost $80 \%$ were patients with the relapsing-remitting form of MS, and there were no patients with clinically isolated syndrome. Mean EDSS was 2.9 (SD: 2.0; range 08 ) with a mean disease duration of 11 years (range: 1-36).

\section{Construct Validity and Internal Structural Validity}

The correlation of each item was higher with the MusiQoL dimension of which it was part than with any other dimension, except for the items of the coping dimension. Floor and ceiling effects can be considered as satisfactory (ceiling effects of RFa and RHCS dimensions were high, 33.0 and 41.1 respectively; Table 2). Dimensions of the MusiQoL showed satisfactory internal consistency (Cronbach's alpha: 0.70-0.92), except for the dimensions of RFa (Cronbach's alpha: 0.67) and RHCS (Cronbach's alpha: 0.53; Table 2).

The overall scalability was satisfactory except for 2 dimensions: the SPT dimension showed an INFIT statistic and a Loevinger coefficient $\mathrm{H}$ outside the acceptable ranges, the RHCS dimension provided a coefficient $\mathrm{H}$ inferior to 0.40 .

\section{External validity}

The concepts covered by the MusiQoL and the SF-36 are not largely overlapping. MusiQoL dimension scores

Table 1 Baseline characteristics of the study population $(n=224)$

\begin{tabular}{|c|c|c|}
\hline Variable & & n (\%) \\
\hline Age, mean (SD), years $(n=224)$ & & $39(10)$ \\
\hline \multirow[t]{2}{*}{$\operatorname{Sex}(n=223)$} & Female & $157(70.4)$ \\
\hline & Male & $66(29.6)$ \\
\hline \multirow[t]{3}{*}{ Poser classification ( $n=223$ ) } & Clinically defined MS & $172(77.1)$ \\
\hline & Laboratory defined MS & $49(22.0)$ \\
\hline & Clinically probable MS & $2(0.1)$ \\
\hline \multirow[t]{3}{*}{ Clinical form of MS $(n=222)$} & Relapsing-remitting & $176(79.3)$ \\
\hline & Secondary progressive & $40(18.0)$ \\
\hline & Primary progressive & $6(2.7)$ \\
\hline Disease duration, mean (SD), range, years & & $\begin{array}{c}10.6(7.2) \\
1-36\end{array}$ \\
\hline EDSS score, mean (SD), range & & $\begin{array}{c}2.9(1.9) \\
0-8\end{array}$ \\
\hline
\end{tabular}

EDSS, Expanded Disability Status Scale; MS, multiple sclerosis; SD, standard deviation. 
Table 2 MusiQoL dimension scale characteristics

\begin{tabular}{|c|c|c|c|c|c|c|c|c|c|}
\hline Dimension (number of items) & Mean (SD) & $\begin{array}{l}\text { MV } \\
(\%)\end{array}$ & $\begin{array}{l}\text { Cronbach's } \\
\text { alpha }\end{array}$ & $\begin{array}{l}\text { IIC, } \\
\text { range }\end{array}$ & $\begin{array}{l}\text { IDV, } \\
\text { range }\end{array}$ & Floor effect, \% & Ceiling effect, \% & $\mathbf{H}$ & INFIT \\
\hline Activities of Daily Living (8) & $57.04(25.96)$ & 1.8 & 0.92 & $0.67-0.80$ & -0.02 to 0.51 & 0.9 & 4.5 & 0.65 & $0.87-1.19$ \\
\hline Psychological Well-Being (4) & $52.95(23.80)$ & 0.4 & 0.88 & $0.70-0.79$ & -0.01 to 0.60 & 2.2 & 4.0 & 0.68 & $0.80-1.12$ \\
\hline Relationships with Friends (3) & $68.61(22.51)$ & 6.3 & 0.80 & $0.59-0.73$ & -0.02 to 0.33 & 1.3 & 13.4 & 0.61 & $0.91-1.12$ \\
\hline Symptoms (4) & $66.45(22.64)$ & 0.9 & 0.70 & $0.43-0.59$ & -0.02 to 0.51 & 0.0 & 11.2 & 0.37 & $0.79-1.20$ \\
\hline Relationships with Family (3) & $82.92(17.82)$ & 1.3 & 0.67 & $0.47-0.56$ & $0.01-0.43$ & 0.0 & 33.0 & 0.44 & $0.98-1.02$ \\
\hline Relationship with Healthcare System (3) & $86.79(16.02)$ & 0.4 & 0.53 & $0.25-0.40$ & -0.02 to 0.23 & 0.0 & 41.1 & 0.30 & $0.82-1.15$ \\
\hline Sentimental and Sexual Life (2) & $73.14(26.52)$ & 16.1 & 0.76 & 0.65 & $0.04-0.38$ & 3.1 & 25.0 & 0.68 & $0.91-1.10$ \\
\hline Coping (2) & $59.31(28.02)$ & 5.4 & 0.75 & 0.60 & $0.13-0.65$ & 3.1 & 15.2 & 0.65 & $0.96-1.01$ \\
\hline Rejection (2) & $83.11(21.62)$ & 7.1 & 0.75 & 0.66 & $0.10-0.43$ & 0.0 & 1.3 & 0.56 & $0.94-1.04$ \\
\hline Index & 70.32 (13.71) & & & & & & & & \\
\hline
\end{tabular}

H Loevinger coefficient; IDV, item discriminant validity; IC, item internal consistency; INFIT item goodness-of-fit (Rasch statistics); MV, percentage of missing values; SD, standard deviation. 
were only moderately correlated with scores of the SF36, except for expected correlation of ADL with physical functioning and vitality; and PWB and COP with $\mathrm{MH}$ $(\mathrm{p}<0.05$; Table 3$)$. The situation was similar when comparing the MusiQoL dimensions with the selfreported 14-symptom scale. ADL on MusiQoL was, at the most, moderately correlated with all components of the 14-symptom scale $(\mathrm{p}<0.05)$, except for 'inability to swallow' (Table 3).

\section{Discriminant validity}

The discriminant validity of MusiQoL was assessed by clinical and sociodemographic characteristics (Tables 4 and 5). There were significant differences based on MS classification in the dimensions of ADL, COP and REJ and the index score $(\mathrm{p}<0.05)$. Patients with secondary progressive MS generally had lower scores (34.5-74.7) than did patients with relapsing-remitting MS (RRMS) (62.4-85.4) or primary progressive MS (31.0-81.3). Differences were also observed when comparing dimension scores by severity rated on the CGI; patients with mild (56.1-88.1) or moderate (47.8-87.4) disease scored higher than those with severe disease (32.6-80.0) for all the domains including the index.

A significant difference was shown in SPT scores in patients with cognitive problems (not including the exclusion criterion of dementia). The time from first symptoms did not correlate with any of the MusiQoL domains. EDSS was only moderately correlated with ADL dimension ( $p>0.01$ ) (Table 4). No significant differences were found according to family history of MS. Patients actually treated and patients treated for the symptoms of relapse did not show significant differences in HRQoL (data not shown).

Women were found to have recorded lower scores than men for PWB. Differences were observed when comparing ADL and SSL scores by family status, with patients living with relatives/family scoring higher for ADL, and couples scoring the highest for SSL and those living with relatives/family $(\mathrm{p}<0.05)$. Patients with a tertiary education had higher scores in the dimensions of ADL, PWB, SPT and RHCS and the index score than did those with a primary or secondary education. Higher scores were also found among students and workers than those who were unemployed in the domains of ADL, PWB, SPT, COP

Table 3 Pearson's correlations between MusiQoL and SF-36, and the 14 self-reported symptom scales

\begin{tabular}{|c|c|c|c|c|c|c|c|c|c|c|}
\hline \multirow[b]{2}{*}{ SF-36 dimensions } & \multicolumn{10}{|c|}{ MusiQoL dimensions } \\
\hline & ADL & PWB & $\mathrm{RFr}$ & SPT & $\mathrm{RFa}$ & RHCS & SSL & COP & REJ & Index \\
\hline Physical functioning & $0.729^{* *}$ & $0.203^{* *}$ & 0.087 & $0.311^{* *}$ & $0.178^{* *}$ & $0.217^{* *}$ & $0.195^{* *}$ & $0.282^{* *}$ & $0.248^{* *}$ & $0.496^{* *}$ \\
\hline Social functioning & $0.515^{* *}$ & $0.347^{* *}$ & $0.212^{* *}$ & $0.441^{* *}$ & $0.178^{* *}$ & $0.198^{* *}$ & $0.275^{* *}$ & $0.373^{* *}$ & $0.222^{* *}$ & $0.541^{* *}$ \\
\hline Role - physical & $0.603^{* *}$ & $0.409^{* *}$ & 0.078 & $0.514^{* *}$ & $0.181^{* *}$ & $0.177^{* *}$ & 0.118 & $0.315^{* *}$ & $0.300^{* *}$ & $0.551 * *$ \\
\hline Role - emotional & $0.443^{* *}$ & $0.443^{* *}$ & 0.099 & $0.527^{* *}$ & 0.115 & 0.126 & $0.164^{*}$ & $0.489^{* *}$ & $0.274^{* *}$ & $0.559 * *$ \\
\hline Mental health & $0.383^{* *}$ & $0.665^{* *}$ & $0.241^{* *}$ & $0.504^{* *}$ & $0.310^{* *}$ & $0.145^{*}$ & $0.389^{* *}$ & $0.654^{* *}$ & $0.465^{* *}$ & $0.760^{* *}$ \\
\hline Vitality & $0.688^{* *}$ & $0.577^{* *}$ & $0.157^{*}$ & $0.550^{* *}$ & $0.258^{* *}$ & $0.214^{* *}$ & $0.354^{* *}$ & $0.532^{* *}$ & $0.364^{* *}$ & $0.699 * *$ \\
\hline Bodily pain & $0.431^{* *}$ & $0.392^{* *}$ & 0.064 & $0.471^{* *}$ & $0.149^{*}$ & $0.180^{* *}$ & $0.157^{*}$ & $0.283^{* *}$ & $0.219^{* *}$ & $0.463^{* *}$ \\
\hline General health & $0.542^{* *}$ & $0.347^{* *}$ & 0.128 & $0.360^{* *}$ & 0.116 & $0.200^{* *}$ & $0.265^{* *}$ & $0.450^{* *}$ & $0.247^{* *}$ & $0.521^{* *}$ \\
\hline 14-symptom scales & ADL & PWB & RFr & SPT & $\mathrm{RFa}$ & RHCS & SSL & COP & REJ & Index \\
\hline Lack of sensation in touch & $-0.295^{* *}$ & -0.057 & 0.070 & -0.112 & 0.068 & 0.126 & -0.160 & 0.062 & -0.019 & -0.100 \\
\hline Lack of sensation in position & $-0.332 * *$ & -0.087 & 0.109 & $-0.192^{*}$ & 0.030 & 0.063 & -0.134 & -0.066 & -0.030 & -0.173 \\
\hline Involuntary body movements & $-0.433^{* *}$ & -0.083 & 0.040 & -0.133 & -0.073 & -0.018 & 0.001 & $-0.213^{*}$ & -0.105 & $-0.249 *$ \\
\hline Vibration in legs or arms & $-0.462^{* *}$ & $-0.185^{*}$ & -0.060 & $-0.274^{* *}$ & -0.083 & -0.092 & -0.083 & $-0.204^{*}$ & -0.167 & $-0.348^{* *}$ \\
\hline Weakness in limbs & $-0.575^{* *}$ & -0.106 & -0.047 & $-0.214^{* *}$ & -0.031 & -0.048 & 0.000 & -0.147 & -0.149 & $-0.242 * *$ \\
\hline Tingling in limbs & $-0.329 * *$ & -0.132 & 0.025 & $-0.246^{* *}$ & -0.040 & -0.071 & -0.010 & -0.067 & -0.127 & $-0.272^{* *}$ \\
\hline Inability to swallow & -0.054 & -0.155 & -0.011 & -0.056 & -0.232 & -0.080 & -0.202 & $-0.279 *$ & -0.052 & $-0.269 *$ \\
\hline Involuntary eye movements & $-0.215^{*}$ & -0.064 & 0.020 & -0.205 & 0.102 & 0.082 & -0.046 & -0.102 & 0.032 & -0.151 \\
\hline Visual problems & $-0.311^{* *}$ & $-0.185^{*}$ & 0.051 & $-0.423^{* *}$ & 0.062 & -0.062 & -0.138 & -0.087 & -0.124 & $-0.321 * *$ \\
\hline Problems concentrating & $-0.425^{* *}$ & $-0.364^{* *}$ & -0.114 & $-0.587^{* *}$ & -0.050 & -0.112 & -0.134 & $-0.323^{* *}$ & $-0.236^{* *}$ & $-0.522 * *$ \\
\hline Difficulty concentrating & $-0.326^{* *}$ & $-0.224^{*}$ & $-0.235^{*}$ & $-0.395^{* *}$ & -0.048 & -0.060 & 0.001 & $-0.234^{*}$ & $-0.388^{* *}$ & $-0.422 * *$ \\
\hline Fatigue & $-0.553^{* *}$ & $-0.222^{* *}$ & -0.075 & $-0.336^{* *}$ & -0.004 & -0.034 & -0.077 & $-0.303^{* *}$ & $-0.184^{*}$ & $-0.373^{* *}$ \\
\hline Urinary incontinence & $-0.404^{* *}$ & -0.118 & -0.074 & $-0.220^{*}$ & -0.078 & -0.019 & $-0.212^{*}$ & -0.058 & -0.050 & $-0.239 *$ \\
\hline Bowel incontinence & $-0.274^{*}$ & -0.190 & -0.020 & $-0.291 * *$ & -0.058 & -0.113 & -0.088 & -0.046 & -0.114 & -0.182 \\
\hline
\end{tabular}

Bold values indicate significant correlation.

${ }^{*} \mathrm{p}<0.05 ;{ }^{* *} \mathrm{p}<0.01$.

$A D L$, activities of daily living; COP, coping; PWB, psychological well-being; REJ, rejection; RFa, relationships with family; RFr, relationships with friends; RHCS, relationship with healthcare system; SPT, symptoms; SSL, sentimental and sexual life. 
Table 4 MusiQoL dimension scores, by clinical characteristics and medical history

\begin{tabular}{|c|c|c|c|c|c|c|c|c|c|c|c|}
\hline \multirow{2}{*}{ Variable } & \multicolumn{11}{|c|}{ MusiQoL dimension } \\
\hline & & $A D L$ & PWB & $\mathrm{RFr}$ & SPT & $\mathrm{RFa}$ & RHCS & SSL & COP & REJ & Index \\
\hline \multicolumn{12}{|l|}{ MS classification } \\
\hline \multirow[t]{2}{*}{ Relapsing-remitting $(n=176)$} & Mean & 63.5 & 54.3 & 69.3 & 67.7 & 83.5 & 87.3 & 73.6 & 62.4 & 85.4 & 72.0 \\
\hline & SD & 24.0 & 23.4 & 22.5 & 22.2 & 17.5 & 16.5 & 25.8 & 26.2 & 20.0 & 13.0 \\
\hline \multirow[t]{2}{*}{ Primary progressive $(n=6)$} & Mean & 31.0 & 50.0 & 76.4 & 65.6 & 81.9 & 80.6 & 72.5 & 57.5 & 81.3 & 65.0 \\
\hline & SD & 18.5 & 22.0 & 13.4 & 28.2 & 25.0 & 17.2 & 37.9 & 31.4 & 25.9 & 9.2 \\
\hline \multirow[t]{3}{*}{ Secondary progressive $(n=40)$} & Mean & 34.5 & 49.1 & 64.9 & 62.9 & 80.1 & 85.0 & 70.5 & 46.9 & 74.7 & 63.3 \\
\hline & SD & 18.9 & 24.7 & 23.7 & 23.2 & 18.7 & 13.7 & 29.7 & 31.8 & 25.6 & 15.9 \\
\hline & $\mathrm{p}$-value & $<0.001$ & 0.435 & 0.402 & 0.475 & 0.569 & 0.464 & 0.854 & 0.009 & 0.021 & 0.011 \\
\hline \multicolumn{12}{|l|}{ Clinical global impression } \\
\hline \multirow[t]{2}{*}{ Mild $(n=118)$} & Mean & 68.7 & 56.1 & 68.2 & 70.8 & 83.0 & 88.1 & 74.0 & 63.5 & 87.0 & 73.2 \\
\hline & SD & 23.9 & 24.0 & 23.8 & 22.1 & 17.8 & 14.3 & 26.0 & 26.4 & 19.8 & 13.2 \\
\hline \multirow[t]{2}{*}{ Moderate $(n=70)$} & Mean & 47.8 & 50.2 & 70.3 & 61.1 & 85.4 & 87.4 & 75.0 & 61.2 & 80.0 & 69.0 \\
\hline & SD & 20.5 & 22.4 & 21.9 & 20.7 & 17.5 & 17.5 & 25.5 & 28.2 & 22.4 & 12.5 \\
\hline \multirow[t]{3}{*}{ Severe $(n=24)$} & Mean & 32.6 & 49.3 & 69.4 & 62.8 & 73.6 & 80.0 & 63.3 & 40.9 & 74.4 & 59.9 \\
\hline & SD & 17.6 & 23.6 & 18.5 & 27.4 & 18.6 & 17.7 & 27.7 & 28.1 & 26.0 & 15.4 \\
\hline & p-value & $<0.001$ & 0.166 & 0.843 & 0.012 & 0.023 & 0.074 & 0.284 & 0.002 & 0.015 & 0.003 \\
\hline \multirow[t]{2}{*}{ MMSE $(n=221)$} & $r$ & 0.29 & 0.15 & 0.05 & 0.23 & 0.04 & 0.12 & 0.09 & 0.15 & 0.25 & 0.26 \\
\hline & p-value & $<0.001$ & 0.029 & 0.488 & 0.001 & 0.575 & 0.085 & 0.204 & 0.028 & $<0.001$ & 0.001 \\
\hline \multirow[t]{2}{*}{ Ambulation Index $(n=220)$} & $r$ & -0.62 & -0.12 & 0.00 & -0.19 & -0.16 & -0.17 & -0.07 & -0.26 & -0.20 & -0.34 \\
\hline & $p$-value & $<0.001$ & 0.075 & 0.985 & 0.006 & 0.022 & 0.011 & 0.378 & $<0.001$ & 0.005 & $<0.001$ \\
\hline \multirow[t]{2}{*}{ EDSS $(n=221)$} & $r$ & -0.63 & -0.14 & 0.02 & -0.25 & -0.04 & -0.18 & -0.06 & -0.29 & -0.26 & -0.35 \\
\hline & $p$-value & $<0.001$ & 0.045 & 0.789 & $<0.001$ & 0.511 & 0.008 & 0.399 & 0.001 & $<0.001$ & $<0.001$ \\
\hline
\end{tabular}

Bold values: $p<0.05$

ADL, activities of daily living; ANOVA, analysis of variance; CIS, clinically isolated syndrome; COP, coping; EDSS, Expanded Disability Status Scale; MMSE, Mini Mental Score Examination; PWB, psychological well-being; r, correlation coefficient; REJ, rejection; RFa, relationships with family; RFr, relationships with friends; RHCS, relationship with healthcare system; SPT, symptoms; SSL, sentimental and sexual life.

and REJ and the index score. For the ADL and SPT domains, patients living with relatives had significantly higher scores than those living alone (Table 5).

\section{Reproducibility}

The number of patients defined as stable between the 2 assessments was 172 according to the physician CGI definition and 190 according to the EDSS. The reproducibility of the MusiQoL domains scores and index score were satisfactory irrespective of the definition of stability, with intraclass correlation coefficients ranging from 0.60-0.91 (Table 6).

\section{Acceptability}

Acceptability was high. Mean (SD) time for completion of the MusiQoL items was 9.8 (11.8) minutes in the patient group as a whole. Mean (SD) time for completion was 9.7 (12.7) minutes in patients with RRMS, 11.6 (7.7) minutes in patients with primary progressive MS and 9.8 (7.4) minutes in patients with secondary progressive MS. The proportion of missing values per dimension was considered to be acceptable, ranging from 0.4 to $7.1 \%$ with the exception of the SSL dimension, which had $16.1 \%$ of values missing.

\section{Discussion}

The MusiQoL study has been a major effort to develop a multidimensional specific HRQoL instrument for patients with MS. It has been developed simultaneously in 1992 patients from 15 countries and in 14 languages [8], using an standardized methodology [18]. It takes into account patients' concerns about QoL, extracting items such as sentimental and sexual life, which patients have described as important for them, and that are not included in other previous instruments $[19,20]$.

Some HRQoL instruments have already been validated in patients with MS in Spain [6,7]. The Spanish version of FAMS was validated in a study that assessed 625 patients with MS recruited in an outpatient clinic setting from 58 hospitals in Spain. Of these, $74 \%$ were patients with RRMS, with a mean age of 37 years, a mean disease duration of 8.8 years and a mean EDSS of 3 points at baseline. The scale showed very good subscale homogeneity comparable to that of the original English version. The Spanish version was not tested for external validity with other HRQoL scales [6].

A transcultural adaptation of the MSQOL-54 has also been validated in a Spanish version. Ten interviews were carried out with 5 men and 5 women with MS, aged 21- 
Table 5 MusiQoL dimension scores, by sociodemographic characteristics

\begin{tabular}{|c|c|c|c|c|c|c|c|c|c|c|c|}
\hline Variable & & ADL & PWB & RFr & SPT & $\mathrm{RFa}$ & RHCS & SSL & $\mathrm{COP}$ & REJ & Index \\
\hline \multicolumn{12}{|l|}{ Sex } \\
\hline \multirow[t]{2}{*}{ Female $(n=157)$} & Mean & 58.4 & 51.0 & 69.8 & 66.0 & 82.7 & 86.8 & 74.6 & 58.8 & 83.8 & 70.5 \\
\hline & SD & 25.4 & 24.0 & 21.0 & 23.0 & 18.4 & 16.0 & 26.2 & 27.8 & 22.1 & 13.6 \\
\hline \multirow[t]{3}{*}{ Male $(n=66)$} & Mean & 54.5 & 58.4 & 66.3 & 68.1 & 83.3 & 86.6 & 70.2 & 61.3 & 81.7 & 70.5 \\
\hline & SD & 26.8 & 21.9 & 25.6 & 21.5 & 16.8 & 16.2 & 27.3 & 27.9 & 20.8 & 13.8 \\
\hline & $\mathrm{p}$-value & 0.316 & 0.033 & 0.311 & 0.541 & 0.821 & 0.915 & 0.292 & 0.552 & 0.538 & 0.998 \\
\hline \multicolumn{12}{|l|}{ Family status } \\
\hline \multirow[t]{2}{*}{ Couple $(n=145)$} & Mean & 52.8 & 50.5 & 68.7 & 64.7 & 83.6 & 86.5 & 75.6 & 60.0 & 82.8 & 69.5 \\
\hline & SD & 26.1 & 22.7 & 21.2 & 22.7 & 18.1 & 16.3 & 23.9 & 28.0 & 22.1 & 13.8 \\
\hline \multirow[t]{2}{*}{ Single $(n=33)$} & Mean & 60.9 & 58.1 & 70.7 & 71.7 & 77.0 & 90.4 & 60.7 & 60.2 & 85.6 & 70.6 \\
\hline & SD & 21.4 & 20.8 & 19.2 & 14.9 & 19.9 & 13.0 & 30.4 & 26.8 & 16.9 & 12.0 \\
\hline \multirow[t]{3}{*}{ With relatives or family $(n=45)$} & Mean & 69.0 & 58.1 & 67.5 & 69.3 & 85.2 & 84.9 & 70.8 & 57.8 & 82.4 & 74.8 \\
\hline & SD & 24.4 & 27.3 & 28.3 & 26.1 & 14.6 & 17.1 & 32.9 & 28.4 & 23.8 & 13.6 \\
\hline & p-value & 0.001 & 0.072 & 0.837 & 0.190 & 0.104 & 0.309 & 0.0048 & 0.899 & 0.776 & 0.214 \\
\hline \multicolumn{12}{|l|}{ Education level } \\
\hline \multirow[t]{2}{*}{ Primary $(n=69)$} & Mean & 49.8 & 47.4 & 68.5 & 62.0 & 83.0 & 88.3 & 75.2 & 57.1 & 82.3 & 67.8 \\
\hline & SD & 27.0 & 20.9 & 21.9 & 23.1 & 18.4 & 15.7 & 25.5 & 29.6 & 24.7 & 13.1 \\
\hline \multirow[t]{2}{*}{ Secondary $(n=82)$} & Mean & 57.1 & 48.9 & 64.8 & 63.5 & 80.1 & 83.2 & 68.6 & 56.3 & 80.3 & 68.1 \\
\hline & SD & 23.9 & 24.6 & 22.5 & 20.6 & 20.2 & 19.1 & 27.2 & 26.2 & 23.4 & 14.0 \\
\hline \multirow[t]{3}{*}{ Tertiary $(n=69)$} & Mean & 64.1 & 63.2 & 73.4 & 74.6 & 85.5 & 89.4 & 76.1 & 66.3 & 87.3 & 75.6 \\
\hline & SD & 25.2 & 21.6 & 22.5 & 22.6 & 14.0 & 11.2 & 27.1 & 26.5 & 14.7 & 12.2 \\
\hline & p-value & 0.005 & $<0.001$ & 0.074 & 0.001 & 0.181 & 0.040 & 0.223 & 0.062 & 0.155 & 0.004 \\
\hline \multicolumn{12}{|l|}{ Employment status } \\
\hline \multirow[t]{2}{*}{ Student $(n=17)$} & Mean & 72.9 & 55.3 & 74.0 & 76.8 & 88.7 & 90.7 & 77.1 & 55.9 & 90.6 & 75.8 \\
\hline & SD & 21.5 & 19.3 & 20.8 & 21.7 & 12.1 & 11.7 & 24.3 & 28.0 & 9.7 & 10.2 \\
\hline \multirow[t]{2}{*}{ Worker $(n=104)$} & Mean & 64.0 & 57.0 & 70.2 & 71.2 & 83.5 & 86.6 & 75.1 & 65.2 & 87.0 & 73.9 \\
\hline & SD & 25.4 & 22.2 & 23.4 & 21.2 & 17.1 & 15.3 & 24.4 & 27.7 & 19.4 & 12.5 \\
\hline \multirow[t]{3}{*}{ Unemployed $(n=102)$} & Mean & 47.7 & 48.9 & 66.4 & 60.3 & 81.3 & 86.2 & 70.4 & 54.6 & 78.2 & 66.2 \\
\hline & SD & 23.6 & 25.1 & 21.8 & 22.4 & 19.3 & 17.3 & 29.2 & 27.0 & 24.1 & 14.0 \\
\hline & $\mathrm{p}$-value & $<0.001$ & 0.047 & 0.319 & $<0.001$ & 0.260 & 0.562 & 0.442 & 0.025 & 0.007 & 0.001 \\
\hline \multicolumn{12}{|l|}{ Housing } \\
\hline \multirow[t]{2}{*}{ Own home $(n=168)$} & Mean & 53.3 & 51.9 & 69.6 & 64.8 & 82.5 & 86.2 & 74.8 & 59.8 & 83.3 & 69.6 \\
\hline & SD & 25.3 & 22.6 & 21.0 & 22.5 & 18.5 & 16.5 & 24.9 & 27.8 & 21.2 & 13.7 \\
\hline \multirow[t]{3}{*}{ With relatives $(n=54)$} & Mean & 70.1 & 57.8 & 66.2 & 72.5 & 84.3 & 88.6 & 66.8 & 59.4 & 83.7 & 74.3 \\
\hline & SD & 23.3 & 25.8 & 26.7 & 21.9 & 15.8 & 14.8 & 32.3 & 28.0 & 22.6 & 12.6 \\
\hline & p-value & $<0.001$ & 0.109 & 0.345 & 0.031 & 0.526 & 0.336 & 0.097 & 0.935 & 0.914 & 0.075 \\
\hline \multirow[t]{2}{*}{ Age } & $r$ & -0.36 & -0.16 & 0.09 & -0.21 & -0.05 & -0.03 & -0.07 & -0.01 & -0.03 & -0.32 \\
\hline & p-value & $<0.001$ & 0.019 & 0.220 & 0.002 & 0.453 & 0.621 & 0.333 & 0.902 & 0.674 & 0.006 \\
\hline
\end{tabular}

Bold values: $p<0.05$.

$A D L$, activities of daily living; ANOVA, analysis of variance; COP, coping; DS, $p<0.05$; NS, not significant; PWB, psychological well-being; $r$, correlation coefficient; $\mathrm{REJ}$, rejection; RF, relationships with family; RFr, relationships with friends; RHCS, relationship with healthcare system; SPT, symptoms; SSL, sentimental and sexual life

54 years, with different education levels and EDSS scores ranging from 1.0 to 8.0. The translated version shows that the Spanish pre-test version is comprehensible and its administration feasible in patients with MS. The psychometric properties must be evaluated in the next phase of the project [7].

The Patient-Reported Indices for Multiple Sclerosis (PRIMUS) is a recently developed scale that comprises three scales for assessing symptoms, activity limitations and QoL in MS. It was translated using a dual-panel process and validated in eight languages, including Spanish. For the Spanish validation, 87 patients were tested, with a mean age of 43 years and 9 years of disease duration at baseline. Forty-seven percent of these patients had RRMS. No data about EDSS at the moment of the study are provided. Most of the tests showed 
Table 6 Reproducibility of MusiQoL scores in patients with stable physician CGI $(n=172)$ and EDSS scores $(n=190)$

\begin{tabular}{lcc}
\hline & $\begin{array}{c}\text { Physician CGI } \\
\text { (n = 172)* }\end{array}$ & $\begin{array}{c}\text { EDSS } \\
(\mathbf{n}=\mathbf{1 9 0})^{* * *}\end{array}$ \\
\hline Activities of Daily Living & 0.89 & 0.90 \\
Psychological Well-Being & 0.81 & 0.90 \\
Relationships with Friends & 0.73 & 0.73 \\
Symptoms & 0.83 & 0.84 \\
Relationships with Family & 0.60 & 0.76 \\
Relationship with Healthcare System & 0.69 & 0.69 \\
Sentimental and Sexual Life & 0.78 & 0.79 \\
Coping & 0.80 & 0.79 \\
Rejection & 0.86 & 0.84 \\
Index & 0.91 & 0.91 \\
\hline
\end{tabular}

CGI, Clinical Global Impression; EDSS, Expanded Disability Status Scale.

good unidimensionality and internal consistency, reproducibility and construct validity in each translated language, including Spanish [21].

Our sample of Spanish patients with MS has similar clinical characteristics (80\% RRMS, disease duration of 10.6 years and mean EDSS of 2.9) to the previously described MS samples [22], so they are perfectly comparable.

This study confirms the process of validation (external validity, internal consistency, reliability and reproducibility) of the Spanish version of the MusiQoL. Our results for validation are satisfactory and similar to those of the international patient sample [8]. This indicates a major strength of the MusiQoL regarding the simultaneous process of validation in different countries.

In the present study, the SF-36 was used for external validation. The SF-36 is a generic questionnaire that has previously been adequately validated in Spanish patients with other health conditions [23-30] and has been used occasionally in patients with MS [31-33]. MusiQoL dimension scores were, in our study, at most moderately correlated with the scores of SF-36, indicating that the two scales do not overlap.

The largest study using the SF-36 for the evaluation of QoL in Spanish patients with MS included 705 patients, $78 \%$ of whom had RRMS, with a mean age of 40.5 years and a median EDSS score of 2.5. These characteristics are similar to those of the patients included in our study and the scores for the eight SF-36 dimensions are also similar. The previous study reported lower physical QoL in patients with higher EDSS scores, but there was no apparent differences in the SF-36 dimensions when stratified by EDSS [31].

We found significant correlations between ADL scores and clinical indices such as EDSS and Ambulation Index, as other authors found [34,35], and the clinical global impression was significantly different in patients with mild or moderate disease, who scored higher than those with severe disease in every MusiQoL domain. These results are similar to the results found in the Spanish FAMS validation study where higher EDSS scores predicted worse scores on all aspect of QoL [8]. PWB score was higher for the male compared to the females. This is a usual result, reported by other authors [36]. These findings emphasize the usefulness of HRQoL instruments, specifically MusiQoL, and indicate the utility of the ADL dimension in reporting the clinical status of patients with MS from their own point of view [37].

The present study had several limitations. The population differs to that in the original study by Simeoni et al [8] as there were no patients with clinically isolated syndrome and only 6 patients with primary progressive MS, preventing the validity of the questionnaire from being evaluated in these groups. Further work could be conducted prospectively in a sample with representative proportions of patients in terms of clinical subtypes of MS. The missing values for most MusiQoL dimensions were considered to be acceptable (range: 0.4-7.1\%), except for the SSL dimension, which had $16.1 \%$ missing values. This highlights that SSL may be an underreported deficit in patients with MS. The external validity was explored by studying relationships between dimensions of MusiQoL and dimensions of SF36, because the SF36 was the single questionnaire available in the needed versions for the initial international validation study; it could be completed by studying correlations of Musiqol and other widespread Spanish MS-specific instruments.

Although MusiQoL could be used with advantage in other Spanish-speaking countries, given its demonstrated acceptable cross-cultural validity [8], small adaptations and further validations would be necessary to ensure reliable application within each of those settings. Even with all these cautions, we point out its potential use as an outcome measure in clinical settings, particularly in multinational clinical trials. 


\section{Conclusions}

The Spanish version of the MusiQoL instrument has been shown to be a valid and reliable instrument for measuring HRQoL in patients with MS. Acceptability was high and time for completion short, and thus MusiQoL constitutes a useful instrument to measure HRQoL in the clinical setting in Spain.

\section{Additional material}

Additional file 1: Table s1a: List of the 31 MusiQoL items (English version). Complete list of the 31 MusiQoL items used in the English version

Additional file 2: Table s1b List of the 31 MusiQoL items (Spanish version). Complete list of the 31 MusiQoL items used in the Spanish version

\section{List of abbreviations}

ADL: Activities of Daily Living; Al: Ambulation Index; BP: Bodily Pain; CGI: Clinical Global Impression; COP: Coping; EDSS: Expanded Disability Status Scale; FAMS: Functional Assessment of Multiple Sclerosis; GH: General Health; HRQoL: Health-Related Quality-of-Life; ICC: Intraclass Correlation Coefficients; IDV: Item Discriminant Validity; IC: Item Internal Consistency; INFIT: Item goodness-of-fit; MAP: Multitrait/multi-item Analysis Program; MH: Mental Health; MMSE: Mini-Mental State Examination; MS: Multiple Sclerosis; MSQOL54: Multiple Sclerosis Quality of Life-54 [questionnaire]; MusiQoL: Multiple Sclerosis International Quality Of Life [questionnaire]; PF: Physical Functioning; PRIMUS: Patient-Reported Indices for Multiple Sclerosis. PWB Psychological Well-Being; QoL: quality-of-life; REJ: Rejection; REP: RoleEmotional Problems; RFa: Relationships with Family; RFr: Relationships with Friends; RHCS: Relationship with the HealthCare System; RPP: Role-Physical Problems; SD: Standard Deviation; SF: Social Functioning; SF-36: 36-Item Short Form [questionnaire]; SPT: Symptoms; SSL: Sentimental and Sexual Life; VIT: Vitality.

\section{Acknowledgements and funding}

This work has been performed using a grant from Merck-Serono S.A. Geneva, Switzerland, an affiliate of Merck KGaA, Darmstadt, Germany. The authors thank Dominic Jack and Joanne Tang, Caudex Medical, Oxford, UK (supported by Merck Serono S.A. - Geneva, Switzerland, an affiliate of Merck KGaA, Darmstadt, Germany), for editorial assistance in the preparation of this manuscript.

The MusiQoL study group of Spain consisted of the following investigators: Hospital Regional Universitario Carlos Haya, Málaga: O Fernández; Hospital de Bellvitge, Barcelona: T Arbizu; Hospital Gregorio Marañón, Madrid: C de Andrés; Hospital Universitario La Fe, Valencia: B Casanova, IB Blasco; Hospital Clínico Universitario, Zaragoza: C Íñiguez; Hospital Central de Asturias, Oviedo: DF Diaz; Hospital Universitario San Cecilio, Granada: M Guerrero; Hospital Universitario Dr. Josep Trueta, Girona: L Ramió; Hospital de Sabadell, Barcelona: MM Igual; Hospital Ramón y Cajal, Madrid: S Calleja; Mutua de Terrassa, Barcelona: IB Ibars; Hospital Arnau de Vilanova, Lleida: P Granes, L Brieva; Hospital del Mar, Barcelona: EM Olivas; Hospital Clínico Universitario de Valencia, Valencia; F Coret; Hospital Virgen de las Nieves, Granada: C Arnal; Complejo Hospitalaria de Jaén, Jaén: MH Pérez; Hospital de Alarcos, Ciudad Real: M Gudín; Hospital del Bierzo, Ponferrada, León: DP Ruiz; Hospital de Cruces, Baracaldo, Bilbao: M Mendibe; Hospital Univ. Virgen de la Arrixaca, Murcia: J Meca; Hospital de Cabueñes, Gijón: S Sanchez; Complejo Hospitalario de Orense, Orense: D Rodriguez.

\section{Author details}

'Institute of Clinical Neurosciences. Service of Neurology. Hospital Regional Universitario Carlos Haya, Avda. Carlos Haya s/n, 29010 Málaga, Spain. ${ }^{2}$ Institute of Clinical Neurosciences. Service of Neurophysiology. Hospital Regional Universitario Carlos Haya, Avda. Carlos Haya s/n, 29010 Málaga, Spain. ${ }^{3}$ Department of Public Health, EA3279 Research Unit, University
Hospital, Boulevard Jean Moulin, 13385 Marseille, France. ${ }^{4}$ Global Medical Affairs Neurology, Merck Serono S.A., 9 Chemin des Mines, 1202 Geneva, Switzerland.

\section{Authors' contributions}

OF participated in study design and implementation in Spain, data collection and interpretation, and the writing of this manuscript. VF participated in the writing and interpretation of this manuscript. KBB and PA were instrumental to the study design, data analysis and interpretation of the results. EVdC participated in trial design and data collection, and interpretation of study findings. $L M, M d M G A, J C A, A L, J C L-M, R B, G L, M G$ and the MusiQoL study group of Spain assisted with data collection. All authors had access to the study data and read and approved the final manuscript.

\section{Competing interests}

OF has received honoraria for serving as a consultant in advisory boards, or chair or speaker in meetings; and for participation in clinical trials and other research projects promoted by Biogen-ldec, Bayer-Schering, Merck Serono, Teva and Novartis. VF, KBB, LM, MdMGA, JCA, AL, JCLM, RB, GL, MG and PA have no competing interests to declare. $\mathbf{E V d C}$ is a salaried employee of Merck Serono S.A. - Geneva, Switzerland.

Received: 26 June 2011 Accepted: 18 October 2011 Published: 18 October 2011

\section{References}

1. Ganz PA: Impact of quality of life outcomes on clinical practice. Oncology 1995, 9:61-65.

2. Solari A: Role of health-related quality of life measures in the routine care of people with multiple sclerosis. Health Qual Life Outcomes 2005, 3:16-16.

3. Quality of Life and Pharmacoeconomics in Clinical Trials Philadelphia: Lippincott-Raven; 1996.

4. Leplege A, Hunt S: The problem of quality of life in medicine. Jama 1997, 278:47-50.

5. McKenna SP: Measuring quality of life in schizophrenia. Eur Psychiatry 1997, 12(Suppl 3):S267-S274.

6. Aymerich M, Guillamon I, Perkal H, et al: Spanish adaptation of the disease-specific questionnaire MSQOL-54 in multiple sclerosis patients. Neurologia 2006, 21:181-187.

7. Chang $\mathrm{CH}$, Cella D, Fernandez $\mathrm{O}$, et al: Quality of life in multiple sclerosis patients in Spain. Mult Scler 2002, 8:527-531.

8. Simeoni M, Auquier $\mathrm{P}$, Fernandez $\mathrm{O}$, et al: Validation of the Multiple Sclerosis International Quality of Life questionnaire. Mult Scler 2008, 14:219-230.

9. Poser CM, Paty DW, Scheinberg $L$, et al: New diagnostic criteria for multiple sclerosis: guidelines for research protocols. Ann Neurol 1983, 13:227-231.

10. McDonald WI, Compston A, Edan G, et al: Recommended diagnostic criteria for multiple sclerosis: guidelines from the International Panel on the Diagnosis of Multiple Sclerosis. Ann Neurol 2001, 50:121-127.

11. Ware JE Jr, Sherbourne CD: The MOS 36-item short-form health survey (SF-36). I. Conceptual framework and item selection. Med Care 1992, 30:473-483.

12. Kurtzke JF: Rating neurologic impairment in multiple sclerosis: an expanded disability status scale (EDSS). Neurology 1983, 33:1444-1452.

13. Hauser SL, Dawson DM, Lehrich JR, et al: Intensive immunosuppression in progressive multiple sclerosis. A randomized, three-arm study of highdose intravenous cyclophosphamide, plasma exchange, and ACTH. N Engl J Med 1983, 308:173-180.

14. Folstein MF, Robins LN, Helzer JE: The Mini-Mental State Examination. Arch Gen Psychiatry 1983, 40:812-812

15. ECDEU Assessment Manual for Psychopharmacology Rockville, MD: US Department of Health and Human Services; 1976.

16. MAP-R for Windows: Multitrait/Multi-ltem Analysis Program - Revised User's Guide Boston, MA: Health Assessment Lab; 1997.

17. Cronbach $\sqcup$ : Coefficient alpha and the internal structure of tests. Psychometrika 1951, 16:297-334.

18. Guillemin F, Bombardier C, Beaton D: Cross-cultural adaptation of healthrelated quality of life measures: literature review and proposed guidelines. J Clin Epidemiol 1993, 46:1417-32. 
19. Nortvedt MW, Riise T, Myhr KM, et al: Reduced quality of life among multiple sclerosis patients with sexual disturbance and bladder dysfunction. Mult Scler 2001, 7:231-235.

20. Schmidt EZ, Hofmann P, Niederwieser G, Kapfhammer HP, Bonelli RM: Sexuality in multiple sclerosis. J Neural Transm 2005, 112:1201-1211.

21. McKenna SP, Doward LC, Twiss J, et al: International development of the patient- reported outcome indices for multiple sclerosis (PRIMUS). Value in Health 2010, 13:946-951.

22. Amato MP, Grimaud J, Achiti I, et al: European validation of a standardized clinical description of multiple sclerosis. J Neurol 2004, 251:1472-1480

23. Alonso J, Regidor E, Barrio G, et al: Population reference values of the Spanish version of the Health Questionnaire SF-36. > Med Clin (Barc) 1998, 111:410-416

24. Wagner AK, Gandek B, Aaronson NK, et al: Cross-cultural comparisons of the content of SF-36 translations across 10 countries: results from the IQOLA Project. International Quality of Life Assessment. J Clin Epidemiol 1998, 51:925-932.

25. Alonso J, Prieto L, Ferrer M, et al: Testing the measurement properties of the Spanish version of the SF-36 Health Survey among male patients with chronic obstructive pulmonary disease. Quality of Life in COPD Study Group. J Clin Epidemiol 1998, 51:1087-1094.

26. Espinosa De Los Monteros MJ, Alonso J, Ancochea J, González A: Calidad de vida en asma: fiabilidad y validez del cuestionario genérico SF-36 aplicado a la población asmática de un área sanitaria. Arch Bronconeumol 2002, 38:4-9.

27. Failde I, Ramos I: Validity and reliability of the SF-36 Health Survey Questionnaire in patients with coronary artery disease. J Clin Epidemiol 2000, 53:359-365.

28. Garcia Ordóñez MA, Mansilla Francisco JJ, Nieto Aragón E, et al: Quality of life associated with the health of patients with HIV infection measured with the Health Questionnaire SF-36. An Med Interna 2001, 18:74-79.

29. Gómez-Besteiro MI, Santiago-Pérez Ml, Alonso-Hernández A, ValdésCañedo F, Rebollo-Alvarez P: Validity and reliability of the SF-36 questionnaire in patients on the waiting list for a kidney transplant and transplant patients. Am J Nephrol 2004, 24:346-351.

30. Ayuso-Mateos JL, Lasa L, Vázquez-Barquero JL, Oviedo A, Díez-Manrique JF: Measuring health status in psychiatric community surveys: internal and external validity of the Spanish version of the SF-36. Acta Psychiatr Scand 1999, 99:26-32

31. Aymerich M, Guillamon I, Jovell AJ: Health-related quality of life assessment in people with multiple sclerosis and their family caregivers. A multicenter study in Catalonia (Southern Europe). Patient Prefer Adherence 2009, 3:311-321.

32. Delgado-Mendilivar JM, Cadenas-Diaz JC, Fernandez-Torrico JM, NavarroMascarell G, Izquierdo G: A study of the quality of life in cases of multiple sclerosis. Rev Neurol 2005, 41:257-262.

33. García-Burguillo MP, Águila-Maturana AM: Energy-saving strategies in the treatment of fatigue in patients with multiple sclerosis. A pilot study. Rev Neurol 2009, 49:181-185.

34. Benedict RH, Wahlig E, Bakshi R, et al: Predicting quality of life in multiple sclerosis: accounting for physical disability, fatigue, cognition, mood disorder, personality, and behavior change. J Neurol Sci 2005, 231:29-34.

35. Yozbatiran N, Baskurt F, Baskurt Z, et al: Motor assessment of upper extremity function and its relation with fatigue, cognitive function and quality of life in multiple sclerosis patients. J Neurol Sci 2006, 246:117-22.

36. Pfaffenberger $\mathrm{N}$, Pfeiffer KP, Deibl M, et al: Association of factors influencing health-related quality of life in MS. Acta Neurol Scand 2006, 114:102-8.

37. Fernández $\mathrm{O}$, Baumstarck-Barrau K, Simeoni MC, Auquier P: Patient characteristics and determinants of quality of life in an international population with multiple sclerosis: Assessment using the MusiQoL and SF-36 questionnaires. Mult Scler 2011.

\section{Pre-publication history}

The pre-publication history for this paper can be accessed here: http://www.biomedcentral.com/1471-2377/11/127/prepub

doi:10.1186/1471-2377-11-127

Cite this article as: Fernández et al: Validation of the spanish version of the multiple sclerosis international quality of life (musiqol) questionnaire. BMC Neurology 2011 11:127.

\section{Submit your next manuscript to BioMed Central and take full advantage of:}

- Convenient online submission

- Thorough peer review

- No space constraints or color figure charges

- Immediate publication on acceptance

- Inclusion in PubMed, CAS, Scopus and Google Scholar

- Research which is freely available for redistribution

Submit your manuscript at www.biomedcentral.com/submit
Biomed Central 